\title{
Diagnostic Validity of Orthopantomogram Compared to Dual Energy X-ray Absorptiometry Scan in Detecting Osteoporosis
}

\author{
D. Prasanna Kumar ${ }^{1} \quad$ S. Jayachandran ${ }^{2} \quad$ N. Thilagavathy ${ }^{1}$
}

\author{
${ }^{1}$ Department of Oral Medicine and Radiology, Adhiparasakthi \\ Dental College and Hospital, Melmaruvathur, Tamil Nadu, India \\ ${ }^{2}$ Department of Oral Medicine and Radiology, Tamil Nadu \\ Government Dental College and Hospital, Chennai, India
}

Address for correspondence D. Prasanna Kumar, MDS, Department of Oral Medicine and Radiology, Adhiparasakthi Dental College and Hospital, Melmaruvathur, Tamil Nadu 603319, India (e-mail: prsnkmr2910@gmail.com).

Ann Natl Acad Med Sci (India) 2021;57:100-107.

\section{Abstract \\ Keywords \\ - osteopenia \\ - osteoporosis \\ - geriatric \\ - bone mineral density \\ - orthopantomography \\ - dual-energy X-ray absorptiometry scan}

Introduction Osteoporosis is one of the most common and rampant metabolic bone disorders among the geriatric, particularly affecting postmenopausal women. Even though resorption tends to occur more rapidly in bones with a higher proportion of trabecular bone (e.g., vertebrae, pelvis, calcaneus), bones with significant cortical bone content also do undergo resorption, for example, mandible. The dental manifestations that may indicate low-bone density include loose teeth, receding gums, and ill-fitting or loose dentures.

Objective To validate the efficacy of orthopantomograms (OPGs) in recognizing bone mineral density (BMD) changes of the mandible using mandibular cortical index $(\mathrm{MCl})$ and substantiate the same with dual energy X-ray absorptiometry (DEXA) scan on femoral neck and spine.

Materials and Methods This cross-sectional study comprised 60 geriatric patients of both genders. All the patients were subjected to panoramic radiographs wherever clinically indicated. The visual analysis was done based on the radiographic appearance of the mandibular cortical border and results were compared with DEXA scan reports, followed by an analysis of three grades of $\mathrm{MCl}$ and BMD statistically.

Results In our study, out of 40 patients in C2 and C3 subgroups, $67 \%$ and $20 \%$ were normal, respectively. The incidence of osteopenia was 33\% in the C2 group and $70 \%$ in the C3 group, whereas Osteoporosis was present only among $10 \%$ of the population in the $\mathrm{C} 3$ group. The difference between the groups are statistically significant $(p=0.01)$. These findings imply that a progressive link exists between BMD and deteriorating cortical morphology.

Conclusion The purpose of this study is that dentists will be able to refer patients to physicians of suspected low BMD, based on incidental findings on panoramic radiographs for further examination. There is a statistically significant correlation present between DEXA and $\mathrm{MCl}$, so the latter can also be used for screening BMD changes. published online April 13, 2021
DOI https://doi.org/

$10.1055 / \mathrm{s}-0041-1724462$ ISSN 0379-038X. (c) 2021. National Academy of Medical Sciences (India).

This is an open access article published by Thieme under the terms of the Creative Commons Attribution-NonDerivative-NonCommercial-License, permitting copying and reproduction so long as the original work is given appropriate credit. Contents may not be used for commercial purposes, or adapted, remixed, transformed or built upon. (https://creativecommons.org/licenses/by-nc-nd/4.0/).

Thieme Medical and Scientific Publishers Pvt. Ltd. A-12, 2nd Floor, Sector 2, Noida-201301 UP, India 


\section{Introduction}

Osteoporosis is one of the most common and rampant metabolic bone disorders among the geriatric, particularly affecting postmenopausal women as a result of unopposed osteoclastic activity and deficiency of estrogen surveillance that promotes increased bone resorption. ${ }^{1}$ Based on the proportion of trabecular bone (TB) and cortical bone (CB) distribution, remodeling pattern varies. Despite the fact that osteoporotic resorption tends to occur more rapidly in bones with a higher proportion of trabecular bone (e.g., vertebrae, pelvis, calcaneus), bones with significant cortical bone content do undergo resorption, for example, mandible. As an aftereffect of increasing average life expectancy, the incidence of osteoporosis is expected to rise concurrently. ${ }^{2}$ The dental manifestations that may indicate low-bone density including loose teeth, receding gums, and ill-fitting or loose dentures.

Osteoporosis is difficult to treat once it progresses to the final stage; hence, early recognition and prevention of the disease is mandatory. It is diagnosed based on the assessment of bone mineral density (BMD) and a subsequent increase in fracture incidence. The World Health Organization (WHO) defines osteoporosis based on T-score; accordingly, T-score of-2.5 or below is stated as osteoporotic, T-score of -1.0 or greater is normal, and T-score between-1.0 and-2.5 is osteopenia. Dual energy X-ray absorptiometry (DEXA) scan is the gold standard investigation to diagnose osteoporosis. ${ }^{3}$ The major impediment in diagnosing osteoporosis using DEXA in a developing nation as India is the limited availability of equipment due to its high cost and lack of substantial epidemiological data pertaining to incidence and prevalence of osteoporosis. ${ }^{4}$ On the other hand, orthopantomogram (OPG), which is mainly used for dental evaluation to scrutinize dental diseases and conditions, is widely available and fairly economical. Therefore, due to its availability and simplicity, it would be beneficial to make use of OPG for assessing individuals with low skeletal bone mineral density (BMD), as the dentists can refer the patient for further examination if required.

Certainly, some examiners did establish a substantial correlation between skeletal BMD and mandible ${ }^{5,6}$ although other research works portrayed contrasting results, demonstrating no such relation. ${ }^{7}$ As per our knowledge, no study has been performed in this province of the south Indian geriatric population to assess osteoporosis using OPG. This study intended to establish a relationship between mandibular cortical integrity on panoramic radiographs and DEXA scan of right and left femoral neck and vertebrae, and to identify whether panoramic radiographs can be used for early identification of osteoporosis-related reduction of bone density among the south Indian geriatric population.

\section{Aims and Objectives}

The aim of the study is to evaluate the diagnostic efficacy of the mandibular cortical index (MCI) in detecting bone marrow density alterations and validate the same by comparing the findings with DEXA scan reports of the south Indian geriatric population.

The objectives of the study are as follows:

1. Obtain complete history and clinically evaluate patients with suspected signs and symptoms of altered BMD in the geriatric population.

2. Radiographic evaluation using orthopantomogram to detect BMD related to osteoporosis and osteopenia in the mandibular cortex.

3. Effectiveness of OPGs using $\mathrm{MCI}$ in diagnosing osteoporosis and osteopenia and comparing the findings with DEXA scan.

\section{Materials and Methods}

A cross-sectional clinical study was conducted on geriatric outpatients reporting to the Department of Oral Medicine and Radiology (OMR), Tamil Nadu Government Dental College and Hospital, Chennai-600 003 (TNGDCH) from June 2016 to November 2017. They were subjected to a thorough clinical examination, details of which were entered into the structured proforma, especially made for the study, and wherever indicated OPG was taken. Informed consent was obtained from all the patients who fulfilled the inclusion and exclusion criteria. The Institutional Ethical Committee approved the study protocol. The study comprises 60 cases in total, among which 35 were male and 25 were female.

The inclusion criteria were male and female patients above 60 years of age and those who were advised OPG for routine dental disorder. The exclusion criteria were medical history positive for bone disease and disorder that influence bone homeostasis.

All the OPGs were visually analyzed using the criteria proposed by Klemetti et al. ${ }^{3}$ The mandibular cortical border near mental foramen on both sides was assessed based on MCI. The $\mathrm{MCI}$ is basically a three-point index (C1-3) that assesses the cortical border of the mandible in OPG using the following criteria.

- C1: The endosteal margin of the mandibular cortex was uniform and well-demarcated on either side (-Fig. 1A).

- C2: The endosteal border displays crescent-shaped defects (lacunar resorption) or appear to form endosteal cortical remains (one to three layers) on one or both sides (-Fig. 1B).

- C3: The cortical layer formed heavy endosteal cortical residues and are porous (-Fig. 1C).
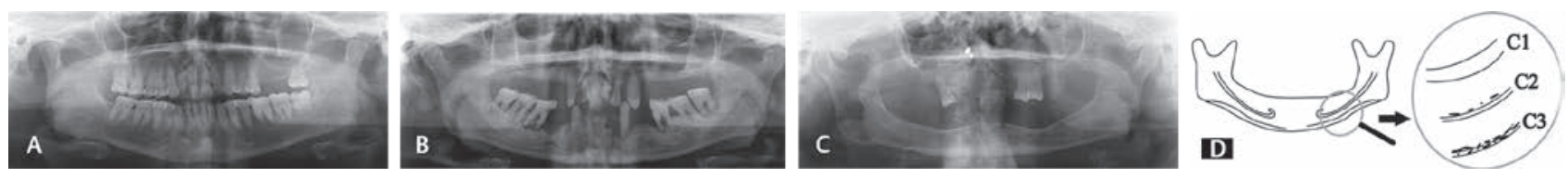

Fig. 1 Different grades of mandibular cortical index: (A) normal mandibular cortex with well-defined borders and uniform thickness, (B) mildly eroded endosteal border, (C) atrophied endosteal border, (D). 
- Fig. 1D depicts three-point index (C1-3) that assesses the cortical border of the mandible in OPG in diagrammatic representation.

$\mathrm{MCI}$ is simple, requires no special equipment for quantification, and is time-saving compared with other densitometric and morphometric indices for which it is considered; additionally, it primarily employs the inferior cortex of the mandible, which is far from alveolar bone and is a component of basal bone which is relatively resilient to resorption due to local factors such as periodontitis. Patients who displayed positive findings related to $\mathrm{MCI}$ were further evaluated for osteopenia or osteoporosis, where the patients were subjected to DEXA analysis to confirm the final diagnosis of their BMD, based on T-score of hip and lumbar spine (L1-L4) with the Discovery DXA densitometer device (Hologic Inc., Bedford, MA, USA).

Based on WHO criteria for osteoporosis, patients who were confirmed to have osteopenia, osteoporosis, or even normal were recorded and results were compared with the OPG already taken and the interpretations were formulated. The results were indexed and evaluated to determine the relationship between the MCI of panoramic radiograph and the BMD acquired from DEXA of the spine (L1-L4) and hip ( - Fig. 2A,B)

\section{Statistical Analysis}

Data were analyzed using SPSS Version 23. As the sensitivity is $100 \%$ and specificity is $69.23 \%$, MCI can very well be used as a screening tool for detecting changes in BMD.

\section{Formulation of Hypothesis}

Null hypothesis: $\mathrm{H}_{0}=$ There is difference in osteoporosis identification by DEXA and MCI index.

Alternate hypothesis $\mathrm{H}_{a}=$ There is no difference in osteoporosis identification by DEXA and MCI index.

$p$ value $<0.05$ is considered as statistically significant.
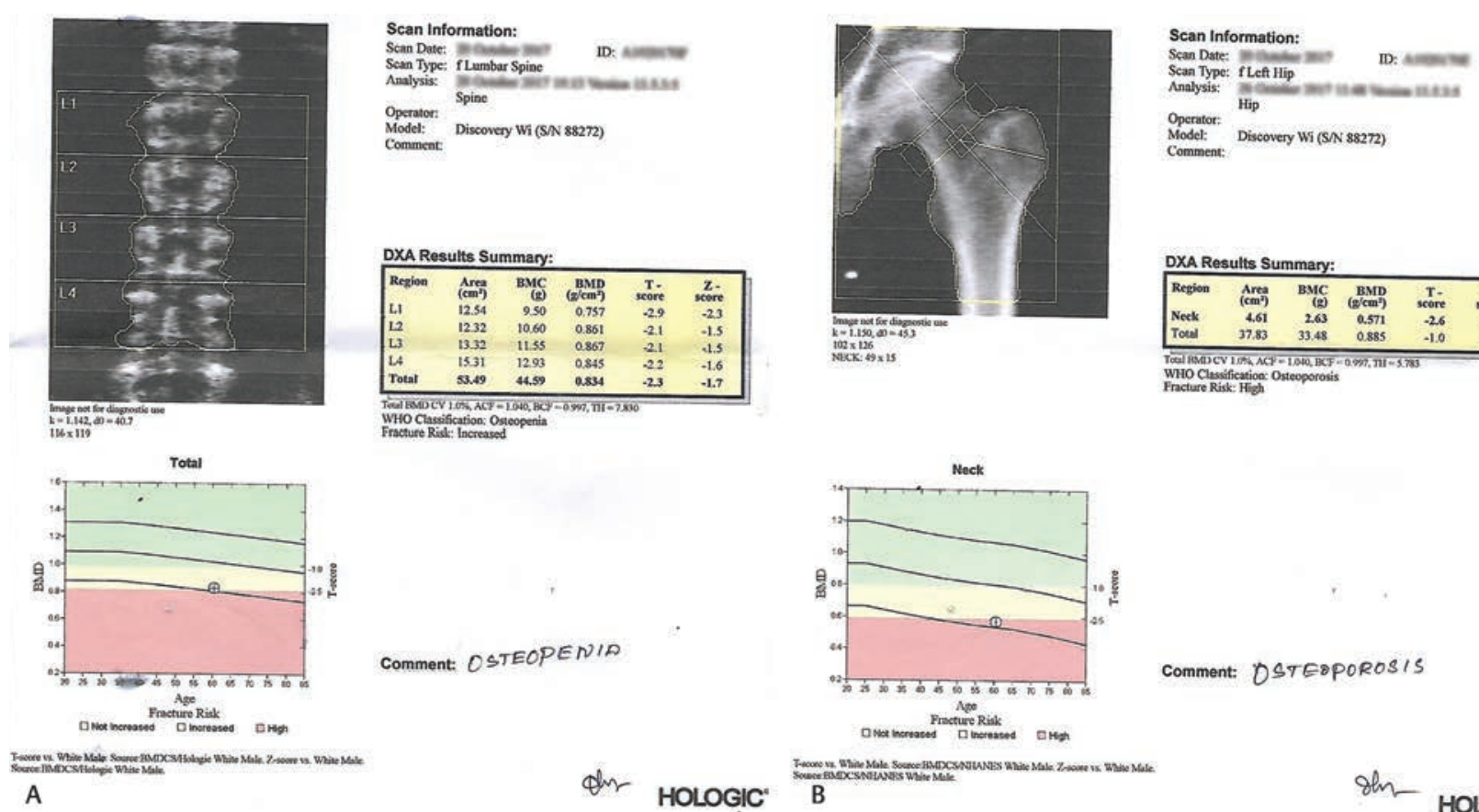

In the present study, a total of 60 cases were included, provisionally evaluated, and clinically examined. Based on OPG findings and $\mathrm{MCI}$, they were categorized as either $\mathrm{C} 1, \mathrm{C} 2$, and C3. Those patients suspected of reduced bone density were subjected to densitometric examination to establish the final diagnosis. The cases diagnosed were then tabulated and analyzed to assess the relationship between the MCI of panoramic radiograph and the BMD obtained from DEXA of hip and lumbar spine (L1-L4).

- Table 1 shows the distribution of the study population according to gender (also see - Fig. 3). In the C1 group, 75\% were males and $25 \%$ were females, whereas in the C2 group, $40 \%$ were females and $60 \%$ were males, and vice versa in the C3 group. This clearly depicts that frequency of females increases as the mandibular cortex gets atrophic.

In - Table 2, DEXA reports showed 60\% normal and $40 \%$ osteopenia in the C2 group (also see - Fig. 4). Higher rates of osteopenia of $70 \%$ were found in the C3 group where only $20 \%$ of the study population was found normal and $10 \%$ was osteoporotic, which clearly shows that $\mathrm{MCI}$ is good enough to assess the changes of BMD.

-Table 3: in the C3 group, the normal population comprised $16.5 \%$ of females and $25 \%$ males (also see - Fig. 5). Osteopenia in the C3 group affected $66.7 \%$ females and $75 \%$ males, while osteoporosis affected only $16.7 \%$ of females in the $\mathrm{C} 3$ group and none in males. The trend correlates with other similar studies, depicting the increased frequency of female involvement.
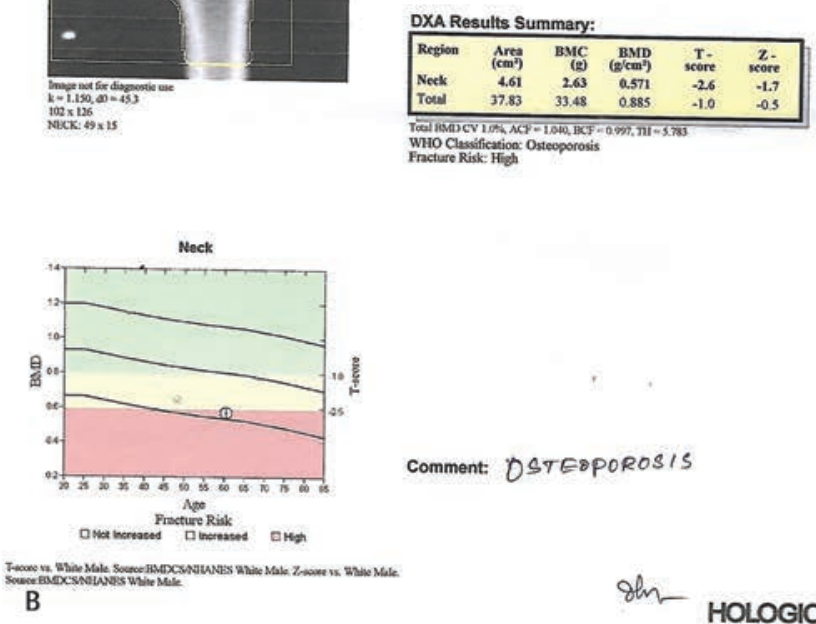

Comment: DSTEOPOROSIS

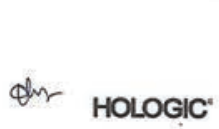

Fig. 2 Bone mineral density measurements with dual-energy X-ray absorptiometry (DEXA): (A) Osteopenia DEXA report, (B) Osteoporosis DEXA report. 
Table 1 Distribution of study population according to gender

\begin{tabular}{|l|l|l|l|l|l|l|}
\hline \multirow{2}{*}{ Gender } & \multicolumn{2}{|l|}{ C1 } & C2 & \multicolumn{2}{l|}{ C3 } \\
\cline { 2 - 7 } & Frequency & Percentage & Frequency & Percentage & Frequency & Percentage \\
\hline Female & 5 & 25.0 & 8 & 40.0 & 12 & 60.0 \\
\hline Male & 15 & 75.0 & 12 & 60.0 & 8 & 40.0 \\
\hline Total & 20 & 100.0 & 20 & 100.0 & 20 & 100.0 \\
\hline
\end{tabular}

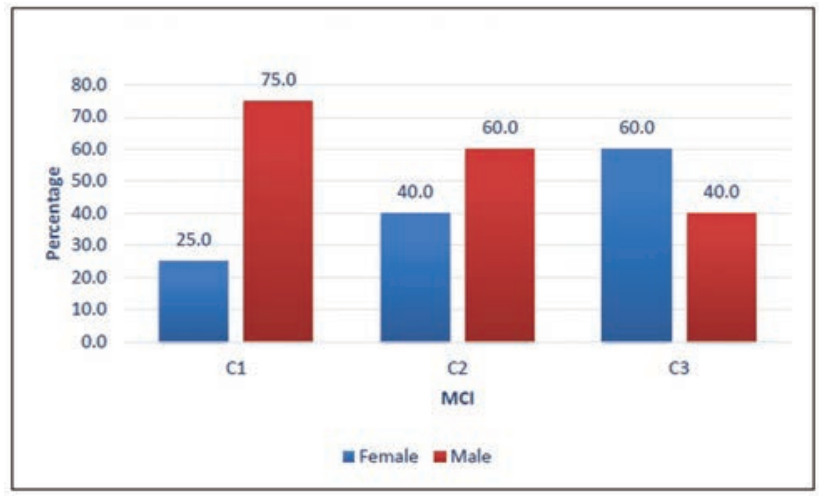

Fig. 3 Distribution of study population according to gender.

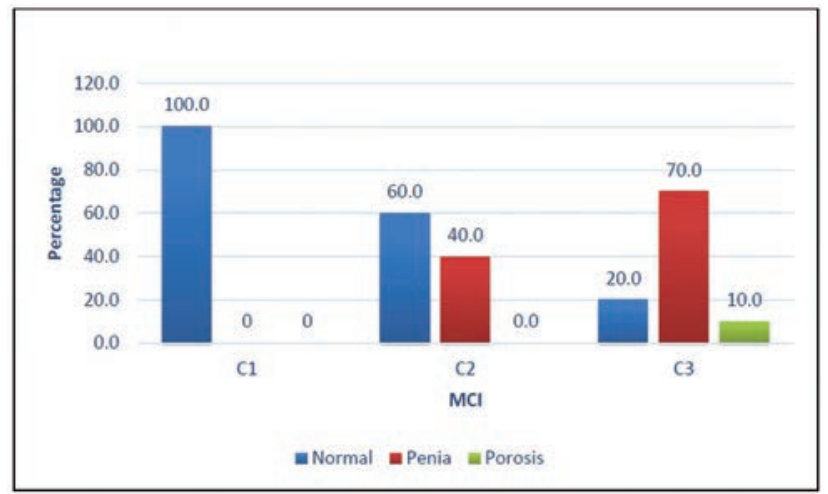

Fig. 4 Distribution of study population according to DEXA report.

Table 2 Distribution according to DEXA report in various groups

\begin{tabular}{|l|l|l|l|l|l|l|}
\hline \multirow{2}{*}{ DEXA report } & C1 & C2 & C3 \\
\cline { 2 - 8 } & Frequency & Percentage & Frequency & Percentage & Frequency & Percentage \\
\hline Normal & 20 & 100.0 & 12 & 60.0 & 4 & 20.0 \\
\hline Osteopenia & 0 & 0 & 8 & 40.0 & 14 & 70.0 \\
\hline Osteoporosis & 0 & 0 & 0.0 & 0.0 & 2 & 10.0 \\
\hline Total & 20 & 100.0 & 20 & 100.0 & 20 & 100.0 \\
\hline
\end{tabular}

Abbreviation: DEXA, dual-energy X-ray absorptiometry.

Table 3 Distribution according to DEXA report in various groups according to gender (C3)

\begin{tabular}{|l|l|l|l|l|}
\hline \multirow{2}{*}{ DEXA report } & Female & Male & Percentage \\
\cline { 2 - 5 } & Frequency & Percentage & Frequency & 25.0 \\
\hline Normal & 2 & 16.7 & 2 & 75.0 \\
\hline Penia & 8 & 66.7 & 6 & 0.0 \\
\hline Porosis & 2 & 16.7 & 0 & 100.0 \\
\hline Total & 12 & 100.0 & 8 & \\
\hline
\end{tabular}

Abbreviation: DEXA, dual-energy X-ray absorptiometry.

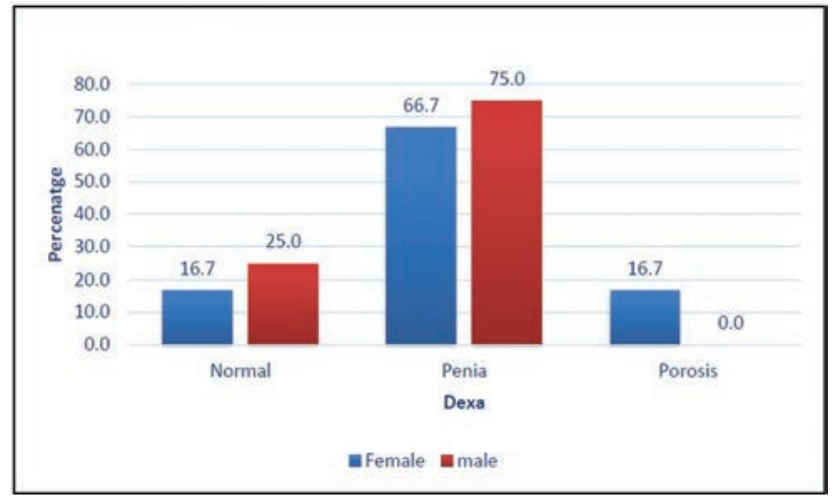

Fig. 5 Distribution according to DEXA reports in C3.
- Table 4 reveals the absence of osteoporosis in both genders in the C2 group (also see - Fig. 6). While $50 \%$ of females were normal, $50 \%$ had an incidence of osteopenia. Among the males, $66.7 \%$ were normal and $33.3 \%$ had osteopenia, which is in concordance with other identical studies showing a good correlation.

- Table 5 clearly shows that, according to MCI, C2 and C3 groups were 67\% and 20\% normal, respectively (also see - Fig. 7). the incidence of osteopenia was $33 \%$ in the C2 group and 70\% in the C3 group, whereas osteoporosis was present only among $10 \%$ of the population in the C3 group. 
104 Diagnostic Validity of Orthopantomogram Compared to DEXA Scan Kumar et al.

Table 4 Distribution according to DEXA report in various groups according to gender (C2)

\begin{tabular}{|l|l|l|l|l|}
\hline \multirow{2}{*}{ DEXA report } & Female & Male & Percentage \\
\cline { 2 - 5 } & Frequency & Percentage & Frequency & 66.7 \\
\hline Normal & 4 & 50.0 & 8 & 33.3 \\
\hline Penia & 4 & 50.0 & 4 & 0 \\
\hline Porosis & 0 & 0 & 0 & 100.0 \\
\hline Total & 8 & 100.0 & 12 & \\
\hline
\end{tabular}

Abbreviation: DEXA, dual-energy X-ray absorptiometry.

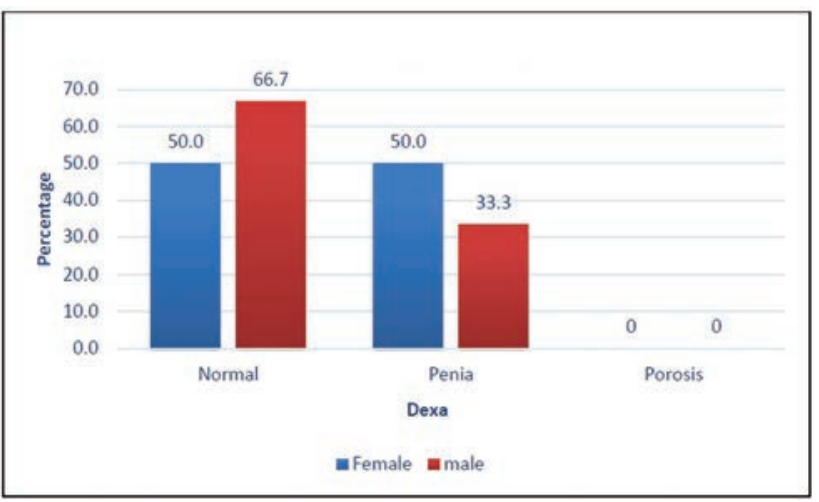

Fig. 6 Distribution according to DEXA reports in C2.

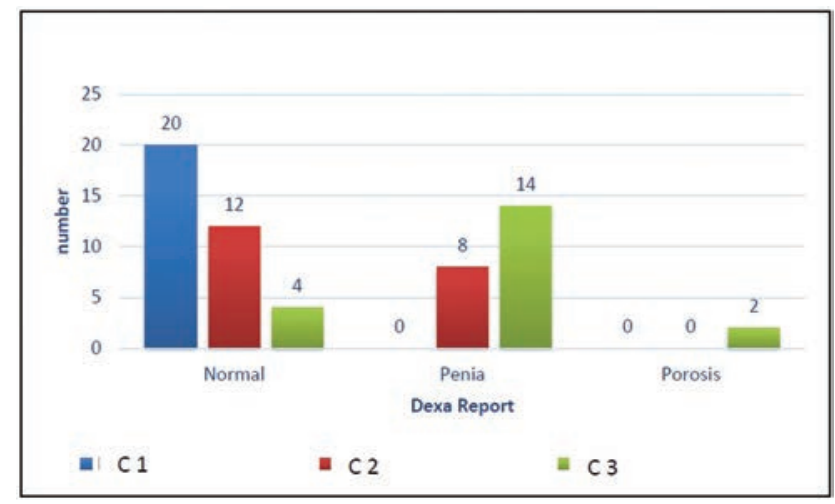

Fig. 7 Comparison of $\mathrm{MCl}$ with DEXA.

Table 5 Comparison of $\mathrm{MCl}$ index with DEXA report

\begin{tabular}{|c|c|c|c|c|}
\hline \multirow[t]{2}{*}{ DEXA report } & \multicolumn{3}{|l|}{$\mathrm{MCl}$} & \multirow[t]{2}{*}{ Total } \\
\hline & C1 & $\mathrm{C} 2$ & $\mathrm{C} 3$ & \\
\hline Normal & $20(100)$ & $12(66.7)$ & $4(20)$ & $36(60)$ \\
\hline Osteopenia & 0 & $8(33.3)$ & $14(70)$ & $22(36.6)$ \\
\hline Osteoporosis & 0 & 0 & $2(10)$ & $2(3.7)$ \\
\hline Total & $20(100)$ & $20(100)$ & $20(100)$ & $60(100)$ \\
\hline Chi-square & \multicolumn{2}{|l|}{28.12} & $p$-Value & $<0.001$ \\
\hline
\end{tabular}

Abbreviations: $\mathrm{MCl}$, mandibular cortical index; DEXA, dual-energy X-ray absorptiometry.

Table 6 Sensitivity and specificity of $\mathrm{MCl}$

\begin{tabular}{|l|l|l|l|l|}
\hline \multicolumn{2}{|l|}{ DEXA OPG cross-tabulation } & Total \\
\hline \multicolumn{2}{|l|}{ Count } & OPG & \\
\cline { 2 - 5 } & Normal & Diseased & 36 \\
\hline \multirow{2}{*}{ DEXA } & Normal & 20 & 16 & 24 \\
\cline { 2 - 5 } & Diseased & 0 & 24 & 60 \\
\hline Total & 20 & 40 & \\
\hline
\end{tabular}

Abbreviations: $\mathrm{MCl}$, mandibular cortical index; OPG, orthopantomogram; DEXA, dual-energy X-ray absorptiometry.

There is a statistically significant correlation present between DEXA and MCI, so the latter can also be used as an alternative to DEXA for measuring BMD.

- Table 6 shows the cross-tabulation between DEXA and OPG, so

The total true positive cases are 24 (A)

Total true negative cases are $36 \quad$ (D)

Total false-positive cases are $16 \quad$ (C)

Total false-negative cases are 0
According to the above,

Sensitivity $=A /(A+B)=100 \%(95 \% C I=85.75-100 \%)$. Specificity $=\mathrm{D} /(\mathrm{C}+\mathrm{D})=69.23 \%(95 \% \mathrm{CI}=54.90-81.28 \%)$. Positive predictive value $(\mathrm{PPV})=\mathrm{A} /(\mathrm{A}+\mathrm{C})=60 \%(95 \% \mathrm{CI}$ $=49.94 \%-69.28 \%$ ).

Negative predictive value $(N P V)=D /(B+D)=100 \%$. Accuracy $=(A+B) /(A+B+C+D)=78.95 \%(95 \% C I$

$=60.08-87.46)$ 
As the sensitivity is cent percent, $\mathrm{MCI}$ can very well be used as a screening tool for detecting changes in BMD.

\section{Discussion}

Osteoporosis is a generalized disorder affecting the skeletal system, which is indicated by reduced bone density and altered microframework of the skeleton, leading to weak bones prone to fractures. Calcium in ionized form has to be maintained by the body within a crucial narrow range for multiple physiologic functions to occur in the vital organs like heart and brain. If this balance is disrupted, either by decreased dietary intake or decreased absorption of calcium, bone tissues undergo resorption to preserve homeostasis. Many lives could be saved, provided osteoporosis is detected at its initial phase. ${ }^{8}$

The WHO describes osteoporosis in terms of T-score of-2.5 or below, T-score of-1.0 or greater as normal, and T-score between-1.0 and-2.5 as osteopenia. DEXA is the gold standard for determining osteoporosis. ${ }^{3}$ Many thirdworld nations do not meet the standards of WHO which advocates 10.6 DEXA machines per million. India hardly meets the dictated standards, with only 0.26 per million in its attic, and conditions are much more worrisome in other south Asian nations with 0.1 per million in Sri Lanka and Pakistan. $^{9}$

In this study, it demonstrates a clear correlation between osteoporosis diagnosis using T-score of DEXA as defined by WHO and OPG radiograph parameters and as per MCI. According to WHO guidelines, BMD is considered to be a vital but not the sole criterion to diagnose osteoporosis. It recognizes other factors that could cause probable bone fractures such as elderly age, hormonal factors, smoking, and falls. ${ }^{10}$ Panoramic radiographs can effectively address the discrepancy between need and feed to facilitate early detection and treatment of osteoporosis, which would reduce associated morbidity and mortality due to its wide availability, simplicity, and, most of all, cost-effectiveness as compared with other advanced imaging modalities. ${ }^{11}$

Multiple indices have demonstrated alterations in BMD using panoramic radiographs. ${ }^{12}$ It could be either radio densitometric or radio morphometric indices; the former deals with quantitative measurement and analysis, whereas latter with morphological or qualitative visual analysis of panoramic radiographs. ${ }^{13} \mathrm{~A}$ radio morphometric index was proposed by Klemetti, which was based on visual analysis of morphological alterations in the cortical border of the mandible called MCI.

Multiple identical studies had established a correlation between mandibular radiographs and reduced BMD indicating osteoporosis. ${ }^{14,15}$ Authors have concluded, demonstrating that the diagnosis of skeletal BMD is possible in up to $95 \%$ by evaluating mandibular cortex. ${ }^{16}$ Scientific sources indicate that the Klemetti index would be beneficial in screening postmenopausal women with osteoporosis. ${ }^{17}$ Some studies concluded claiming that a large sample size is needed to evaluate the efficacy of $\mathrm{MCI}$ in BMD studies, while in other studies, it was not adequately accurate for evaluation. ${ }^{18}$ To date, no study has been performed on association of aforementioned indices with mandibular BMD levels in the south Indian geriatric population.

As the risk of osteoporosis increases with a related increase in age, the present study was conducted on geriatric subjects. ${ }^{19}$ The lower age limit in this study was set as the sixth decade, as the bone density starts to reduce in about the third decade of life. ${ }^{20}$ Among the evaluated geriatric, $60 \%$ of patients had normal bone density and the rest $40 \%$ had reduced BMD. This is true with other studies, which also expressed the irrefutable statistics of a higher incidence of osteoporosis associated with elderly patients.

The present study was in concordance to similar studies where females reported a higher incidence of osteoporosis and osteopenia. Menopause in women leads to rapid bone loss in females compared with men who possess a progressive recession of sex hormones. ${ }^{21}$ In parallel research works, a significant correlation between BMD and the mandibular cortex was reported. ${ }^{22}$

In the present study, 2 (3.7\%) patients had osteoporosis, 22 (36.6\%) osteopenia, and 36 (60\%) patients had normal findings, based on the DEXA report. Among this, 3.7\% diagnosed with osteoporosis included females only. In an identical study, 51.5\% of all included women had osteoporosis, while $7.8 \%$ of all included men had osteoporosis, based on DEXA. ${ }^{23}$

The efficacy of the aforementioned indices is determined by its sensitivity and specificity as compared with DEXA results. The sensitivity and specificity of one of the studies were $77 \%$ and $53 \%$, respectively. ${ }^{24}$ In a different study, the specificity and sensitivity were $88 \%$ and $100 \%$, respectively. ${ }^{25}$ In another similar study, when the threshold for a reduced BMD was set at a T-score of-1.0, a sensitivity of $73.0 \%$ and a specificity of $49 \%$ were found, which employed DEXA on the lumbar spine and the proximal femur to determine BMD. ${ }^{26}$ In our study, the T-score threshold was set at-2.5 as defined by WHO. This included both geriatric men and women, where $\mathrm{MCI}$ was used as a diagnostic criterion, and it was compared with gold standard, that is, DEXA results, which yielded a sensitivity and a specificity of more than $98 \%$ and $69 \%$, respectively.

It is apparent that $\mathrm{MCI}$ is the most evaluated variable in the mandible related to osteoporosis. Likewise, $\mathrm{MCI}$ was found to be a reliable index in detecting a decrease in BMD from this study. It is evident from the present study that patients with low BMD exhibited distinct delicate porous mandibular cortices, and the findings correlate with other studies. As MCI is a qualitative and objective index, it does not require precise identification of panoramic landmarks and accurate analysis; as a result of which, some studies do proclaim contrasting results to the present study, demonstrating no correlation between $\mathrm{MCI}$ and BMD. ${ }^{27}$

Some authors hypothesized that as there would be differences in the width of the mandible between Asian and British postmenopausal women, the crude statistics of cortical width could not be applied directly for detecting osteoporosis. ${ }^{28}$ In addition to this, no authorized data are available that recommends the threshold values for the various mandibular 
indices which could be employed on the Indian population. $\mathrm{C} 1, \mathrm{C} 2$, and $\mathrm{C} 3$ was the proportional distribution of MCI categorization, with 20 in each group. With minimal variance between BMD and $\mathrm{MCI}$, and the proportion of categorization nearly complementing, the difference between groups was statistically significant $(p=0.01)$. These findings imply that a progressive link exists between BMD and deteriorating cortical morphology.

\section{Conclusion}

Osteoporosis is preventable and curable when detected early; thus, detection of BMD changes at its initial stages is of prime importance. Among the elderly, the dentist is the most frequently visited doctor. As panoramic radiographs are the unanimously employed imaging modality, they offer the dentist an opportunity to screen sizable population. ${ }^{29}$ Based on this study, sensitivity for the $\mathrm{MCI}$ was found to be more than $95 \%$. With this, we can suggest the orthopedic community to utilize OPG as their screening tool, as this turns out to be a simple, quick and devoid of any special instrument requirements and, most importantly, might support the financially compromised subjects of our community. By utilizing these, dentists might be able to scrutinize high-risk geriatric patients with osteoporosis who might need immediate medical attention for further assessment and treatment by evaluating dental radiographs. Further studies with a larger sample of greater ethnic diversity are needed to better elucidate whether dentists could detect early warning signs of osteoporosis risk.

\section{Limitations of the Study}

However, there are a few limitations to the current study. Because of limited resources, other risk factors such as backcountry or urbane, literacy status, other data like height and shape of the alveolus, number of teeth present and, most of all, female participants' estrogen status were not included and were assumed to be normal. In addition to this, the MCI index due to its substantial intra- and interexaminer variability and its poor reproducibility holds limited validity. ${ }^{30}$ As bone is a heterogeneous structure, BMD varies in each area. Thus, BMD of spine and femur neck alone cannot be solely considered the standard. Accordingly, other markers such as serum and urinary markers should be considered in assessing general bone status, which was not included in the present study. Many factors affect the radiographic findings, for example, standardization of head positioning, projection, optimized radiation dose, and anatomic variability. However, for MCI only, minor discrepancy was reported to be caused by positioning error and operatory error. ${ }^{31}$ Few similar studies have proclaimed fair reproducibility with $\mathrm{MCl}{ }^{32,33}$ The orthopedic community can still look forward to this study, as this still prevails in displaying findings concerning age in addition to BMD.

MCI could very well be utilized as an effective economical screening tool in detecting occult BMD changes before opting for direct DEXA scan. Less developed nation as us, where DEXA scan facilities are not readily available for all, dentists' contribution becomes vital. OPG could very well reduce unnecessary referral to distant DEXA facility centers. Moreover, in detecting bone density changes by dentists, it has dual-edge advantage, as the prognosis is better. It should be emphasized that OPG markers and oral cavity changes can only serve as a screening tool, whereby a confirmatory test with DEXA can be prescribed for osteoporosis detection.

\section{Conflict of Interest}

None declared.

\section{References}

1 Sabzwari S, Azhar G. Ageing in Pakistan-A new challenge. Ageing Int 2010;36(4):423-427

2 Horner K, Devlin $\mathrm{H}$. The relationships between two indices of mandibular bone quality and bone mineral density measured by dual energy X-ray absorptiometry. Dentomaxillofac Radiol 1998;27(1):17-21

3 Lundström A, Jendle J, Stenström B, Toss G, Ravald N. Periodontal conditions in 70-year-old women with osteoporosis. Swed Dent J 2001;25(3):89-96

4 Delmas PD, Marin F, Marcus R, Misurski DA, Mitlak BH. Beyond hip: importance of other nonspinal fractures. Am J Med 2007;120(5):381-387

5 Khosla S, Amin S, Orwoll E. Osteoporosis in men. Endocr Rev 2008;29(4):441-464

6 Richards JB, Kavvoura FK, Rivadeneira F, et al; Genetic Factors for Osteoporosis Consortium. Collaborative meta-analysis: associations of 150 candidate genes with osteoporosis and osteoporotic fracture. Ann Intern Med 2009;151(8):528-537

7 Center JR, Bliuc D, Nguyen TV, Eisman JA. Risk of subsequent fracture after low-trauma fracture in men and women. JAMA 2007;297(4):387-394

8 Raisz LG. Pathogenesis of osteoporosis: concepts, conflicts, and prospects. J Clin Invest 2005;115(12):3318-3325

9 Varthakavi PK, Joshi AS, Bhagwat NM, Chadha MD. Osteoporosis treatment in India: Call for action. Indian J Endocrinol Metab 2014;18(4):441-442

10 van den Bergh B, Karagozoglu KH, Heymans MW, Forouzanfar T. Aetiology and incidence of maxillofacial trauma in Amsterdam: a retrospective analysis of 579 patients. J Craniomaxillofac Surg 2012;40(6):e165-e169

11 Kumar P, Singh SV, Aggarwal H, Alvi HA. Call for detection of osteoporosis in India-can a dentist help? Indian J Endocrinol Metab 2014;18(6):871

12 Andre F, Paulo T, Claudia M, Nilse S. Correlation between seven panoramic radiomorphometric indices and bone mineral density in postmenopausal women. Oral Surg Oral Pathol Oral Radiol Oral Endodont 2010;109(3):449-456

13 Ishii K, Taguchi A, Nakamoto T, et al. Diagnostic efficacy of alveolar bone loss of the mandible for identifying postmenopausal women with femoral osteoporosis. Dentomaxillofac Radiol 2007;36(1):28-33

14 Amorim MA, Takayama L, Jorgetti V, Pereira RM. Comparative study of axial and femoral bone mineral density and parameters of mandibular bone quality in patients receiving dental implants. Osteoporos Int 2007;18(5):703-709

15 Devlin H, Allen P, Graham J, et al. The role of the dental surgeon in detecting osteoporosis: the OSTEODENT study. Br Dent J 2008;204(10):E16-E16

16 Taguchi A. Triage screening for osteoporosis in dental clinics using panoramic radiographs. Oral Dis 2010;16(4):316-327

17 Bhatnagar S, Krishnamurthy V, Pagare SS. Diagnostic efficacy of panoramic radiography in detection of osteoporosis in post-menopausal women with low bone mineral density. J Clin Imaging Sci 2013;3:23 
18 Marandi S, Bagherpour A, Imanimoghaddam M, Hatef M, Haghighi A. Panoramic-based mandibular indices and bone mineral density of femoral neck and lumbar vertebrae in women. J Dent (Tehran) 2010;7(2):98-106

19 Nagler RM, Hershkovich O. Relationships between age, drugs, oral sensorial complaints and salivary profile. Arch Oral Biol 2005;50(1):7-16

20 Balcikonyte E, Balciuniene I, Alekna V. Panoramic radiographs in assessment of the bone mineral density. Stomatologija 2004;6:17-19

21 Mundy GR. Osteoporosis: pathophysiology and nonpharmacological management. Best Pract Res Clin Rheumatol 2001;15(5):727-745

22 Watanabe PC, Oliveira TM, Monteiro SA, et al. Morphodigital study of the mandibular trabecular bone in panoramic radiographs. Int J Morphol 2007;25:875-880

23 Kanis JA, Johnell O, Oden A, Jonsson B, De Laet C, Dawson A. Risk of hip fracture according to the World Health Organization criteria for osteopenia and osteoporosis. Bone 2000;27(5):585-590

24 Mahl CRW, Licks R, Fontanella VRC. Comparison of morphometric indices obtained from dental panoramic radiography for identifying individuals with osteoporosis/osteopenia. Radiol Bras 2008;41:183-187

25 Gaur B, Chaudhary A, Wanjari PV, Sunil M, Basavaraj P. Evaluation of panoramic Radiographs as a screening tool of osteoporosis in post menopausal women: a cross sectional study. J Clin Diagn Res 2013;7(9):2051-2055

26 Sutthiprapaporn P, Taguchi A, Nakamoto T, et al. Diagnostic performance of general dental practitioners after lecture in identifying post-menopausal women with low bone mineral density by panoramic radiographs. Dentomaxillofac Radiol 2006;35(4):249-252

27 Drozdzowska B, Pluskiewicz W. Longitudinal changes in mandibular bone mineral density compared with hip bone mineral density and quantitative ultrasound at calcaneus and hand phalanges. Br J Radiol 2002;75(897):743-747

28 Taguchi A, Ohtsuka M, Nakamoto T, et al. Identification of post-menopausal women at risk of osteoporosis by trained general dental practitioners using panoramic radiographs. Dentomaxillofac Radiol 2007;36(3):149-154

29 Kumar P, Singh SV, Aggarwal H, Alvi HA. Call for detection of osteoporosis in India-can a dentist help? Indian J Endocrinol Metab 2014;18(6):871

30 Jowitt N, MacFarlane T, Devlin H, Klemetti E, Horner K. The reproducibility of the mandibular cortical index. Dentomaxillofac Radiol 1999;28(3):141-144

31 Taguchi A, Tanimoto K, Suei Y. The estimation of the radiomorphometric indices of the mandible using panoramic radiography. Dent Radiol 1993;33:309-316

32 Halling A, Persson GR, Berglund J, Johansson O, Renvert S. Comparison between the Klemetti index and heel DXA BMD measurements in the diagnosis of reduced skeletal bone mineral density in the elderly. Osteoporos Int 2005; 16(8):999-1003

33 Taguchi A, Tsuda M, Ohtsuka M, et al. Use of dental panoramic radiographs in identifying younger postmenopausal women with osteoporosis. Osteoporos Int 2006;17(3):387-394 